\title{
Intra-articular hyaluronic acid vs platelet-rich plasma in the treatment of hip osteoarthritis
}

\author{
Luca Di Sante ${ }^{1}$, Ciro Villani ${ }^{3,4}$, Valter Santilli'i, ${ }^{1,2}$, Massimo Valeo ${ }^{3,4}$, Emmalisa Bologna $^{2}$, \\ Luca Imparato ${ }^{4}$, Marco Paoloni ${ }^{1}$, Annamaria Iagnocco ${ }^{5}$
}

${ }^{1}$ Physical Medicine and Rehabilitation Unit, AziendaPoliclinico Umberto I, ${ }^{2}$ Department of Physical Medicine and Rehabilitation, Sapienza Università di Roma, ${ }^{3}$ Orthopaedic Clinic Azienda Policlinico Umberto I, ${ }^{4}$ Department of Orthopedic Science, Sapienza Università di Roma, ${ }^{5}$ Dipartimento Scienze Cliniche e Biologiche - Reumatologia, Università degli Studi di Torino, Tourin, Italy

\begin{abstract}
Aim: To compare the efficacy of ultrasound-guided intra-articular (IA) treatment with platelet-rich plasma (PRP) versus viscosupplementation (hyaluronic acid [HA]) in hip osteoarthritis. Material and methods: A total of 43 patients affected by monolateral severe hip osteoarthritis (OA) were included in the study. Patients were randomized to receive either intra-articular PRP (3 ml) or HA (30 mg/2 ml; 1,000-2,900 kDa), 3 injections in total-1/week. Clinical assessments for each patient were made at baseline (T0), 4 (T1), and 16 weeks (T2) of follow-up. The primary efficacy outcome was pain reduction as measured by VAS and by WOMAC pain subscale. Results: Data analysis revealed that, compared to T0, in the PRP-treated group VAS scores significantly decreased at T1 but not at T2, thereby indicating an early effect on pain which was not maintained at a longer term follow-up. In the HA group a significant decrease of both VAS and WOMAC values was registered only between T0 and T2. Conclusions: Intra-articular PRP had an immediate effect on pain that was not maintained at longer term follow-up when, on the contrary, the effects of intra-articular HA were evident.
\end{abstract}

Keywords: hip osteoarthritis, Platelet-Rich Plasma, Hyaluronic Acid, ultrasound-guided injection.

\section{Introduction}

Osteoarthritis (OA) is a degenerative disease of the synovial joints with global articular involvement, including hyaline cartilage and subchondral bone. It is the most common cause of chronic pain in the elderly. Particularly, hip $\mathrm{OA}$ is one of the main causes of functional disability and joint pain in adults older than 55 years [1-5]. Current treatment strategies with either non-pharmacologic or pharmacologic therapies aim to reduce pain and physical disability and, possibly also, to limit structural deterioration of the affected joints. In the last decade the use of intra-articular (IA) injections of hyaluronic acid (HA) has become more and more popular, and recently a number

Received 05.08.2016 Accepted 17.09.2016

Med Ultrason

2016, Vol. 18, No 4, 463-468

Corresponding author: Annamaria Iagnocco, MD

Sapienza Università di Roma, Rome, Italy

Viale del Policlinico 155 - 00161 Rome, Italy

Phone: +39-6-49973062

E-mail: annamaria.iagnocco1@gmail.com of studies have addressed the efficacy of this therapeutic intervention on pain and function in hip OA [6,7]. IA injections have many effects: primarily restoration of elastic and viscous properties of synovial fluid, but also anti-inflammatory and anti-nociceptive effects, as well as normalization of hyaluronan synthesis by synoviocytes [8].

Current research in the field is aimed at investigating new methods for stimulating the repair of damaged cartilage. The most recent acquisitions regarding tissue biology highlighted the complex regulation of growth factors (GFs) for the normal tissue structure and the reaction to tissue damage. In addition, the influence of these growth factors on cartilage repair has been widely investigated in vitro and in vivo [9-12].

Platelet-rich plasma (PRP) therapy is a feasible, minimally invasive and relatively inexpensive treatment that allows a natural concentrate of autologous growth factors to be obtained from the blood. This therapy is widely experimented in different fields of medicine to test its potential role to enhance tissue re-generation [13-17]. In a recent non-controlled prospective trial, the safety, toler- 
ability and efficacy of PRP IA injections have been the subject of a preliminary report on 40 hip OA patients at 7 weeks' and 6 months' follow-up [18]. More recently, ultrasound-guided injection of platelet-rich plasma and HA were used separately and in combination in hip OA patients in a randomized controlled study [19]. Moreover, the efficacy of ultrasound-guided intra-articular injections of PRP versus HA was assessed in another study focused on hip OA [20].

Based on the previously reported researches in the field, the aim of the present study is to test the efficacy of PRP IA therapy as compared to HA IA treatment in terms of pain relief and functional recovery in a population of hip OA patients.

\section{Material and methods}

Consecutive patients of both genders with a diagnosis of hip OA, according to American College of Rheumatology criteria [21], were enrolled in the study. Exclusion criteria were the following: I and IV Kellgren and Lawrence scores [22]; clinical evidence of hip joint instability; previous open or arthroscopic hip surgery; history of systemic or local infectious, neoplastic and/or other rheumatic diseases; haematological diseases (coagulopathy); severe cardiovascular diseases; infections; immunodepression; patients in therapy with antiplatelet drugs; and patients with $\mathrm{Hb}$ values $<11 \mathrm{~g} / \mathrm{dl}$ and platelet values $<150,000 / \mathrm{mmc}$.

The study protocol was approved by the local Ethics Committee. The experimental protocol was carefully explained to the patients participating in the study, and their informed consent was obtained by signing a detailed form in accordance with the Declaration of Helsinki. Participants were randomly assigned to either the HA group or PRP group by an independent person who selected a sealed envelope 30 minutes before the intervention was due to start.

\section{Clinical outcome measures}

Self-rated pain intensity at the moment of the evaluation was measured on a $10-\mathrm{cm}$ horizontal visual-analogue scale (VAS), with $0 \mathrm{~cm}$ labeled as "no pain" and 10 $\mathrm{cm}$ labeled as "worst pain I have ever had". The Italian version of the Western Ontario and McMaster Universities (WOMAC) OA index [23], a self-assessment multidimensional instrument that evaluates 17 functional activities, 5 pain-related activities, and 2 joint stiffness categories in 3 different subscales, was used to measure dysfunction and pain.

\section{Platelet-rich plasma preparation}

PRP was obtained following the instructions for the use of the Regen Kit ${ }^{\circledR}$ in the preparation of the Autologous Platelet Gel. The procedure entailed a $8-\mathrm{ml}$ venous blood sample for each hip treated. A complete peripheral

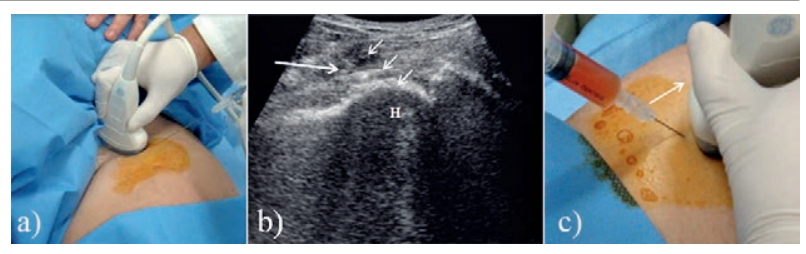

Fig 1. a) Free-hand technique with US probe placed parallel to the femur neck; b) sagittal US showing synovium/capsule (long arrow), the needle situated in the articulation (three short arrows), and the femoral head (H); c) PRP ultrasound-guided IA injections

blood count was also collected at the time of the initial blood donation. The samples were centrifuged twice at $3100 \mathrm{rpm}$ (for $9 \mathrm{~min}$ ) in order to produce $4 \mathrm{ml}$ of PRP. After turning the test-tube upside down repeatedly, so as to achieve a homogeneous distribution, we linked the testtube to the transferral and aspirated the content.

All procedures were performed in the same laboratory setting. All of the open procedures were performed in an A-class sterile hood. Randomly one PRP unit was sent to the laboratory for quality analysis (platelet count and bacteriological test). The total number of platelets $/ \mathrm{ml}$ in the PRP without leukocytes represented a mean increase of around $100-150 \%$ compared with whole blood values.

\section{Therapeutic Procedure}

By using a computer-generated 1:1 allocation sequence, patients were randomized to either receiving $\mathrm{Na}-\mathrm{HA}(30 \mathrm{mg} / 2 \mathrm{ml}$ of HA with molecular weight 1,000 to $2,900 \mathrm{kDa}$ ) or PRP (3 ml) injections (3 injections in total $-1 /$ week). For patients who satisfied the inclusion criteria the procedure entailed a $8-\mathrm{ml}$ venous blood sample (see above). Ultrasound (GE Healthcare, Logiq P5 pro) was used as guidance for performing the IA procedure in all cases [24]. The injection was performed under sterile conditions by means of a $3.5 \mathrm{MHz}$ convex probe. The patients laid supine with the hip in $15^{\circ}-20^{\circ}$ internal rotation, and previously to the injection, the hip region was scanned in order to localize the most relevant local landmarks such as the femoral neurovascular bundle, the femoral neck, the hip joint capsule, and the anterior synovial recess (fig 1). The probe was then aligned with the long axis of the femoral neck, including the acetabulum and the femoral head and, by a freehand technique, a 20 -gauge $(9 \mathrm{~cm})$ spinal needle was then advanced under direct ultrasound guidance into the anterior synovial recess at the junction of the femoral head and neck. Once the needle touched the bone it was retracted by $1 \mathrm{~mm}$ and a synovial fluid aspiration was performed previously to the IA injection in order to decrease dilutions of the injected therapy [25]. Subsequently the injection was performed under sonographic guidance. As an additional confirmation of the correctly performed procedure and 
IA therapy placement, a distension of the joint capsule was registered. After a few minutes of rest, the patients were allowed to walk and leave the clinic. They were also advised to rest until the next morning and during the follow-up period (16 weeks); the use of any anti-inflammatory or analgesic medication was not allowed. In addition, all patients were monitored for any side effects due to the IA injections.

\section{Follow-Up}

All patients underwent clinical evaluation at baseline (T0), 4 weeks (T1) and 16 weeks (T2) after the therapeutic procedure (fig 2). As a primary outcome measure, we considered pain reduction as measured by VAS. As secondary outcome measures, we considered pain reduction as measured by the WOMAC pain subscale (WOMAC A), and functional improvement as measured by WOMAC joint stiffness (WOMAC B) and disability (WOMAC C) subscales. All questionnaires were answered before the physical examination performed in the out-patient clinic.

\section{Statistical analysis}

Sample size was calculated for the primary end point. Assuming from a pilot study a mean difference from baseline of 3-points of the VAS score at T2 with an $\alpha$ error of 0.05 , a $\beta$ error of 0.2 the minimum sample size was 17 for each group. Assuming a dropout of 15\%, 20 patients per group were required. The statistical analysis was performed using the MedCalc version 10.2.0.0 for Windows. All primary and secondary outcome analyses were performed according to the principle of intentionto-treat [22]. The chi-square or 2-sample t-tests were ap-

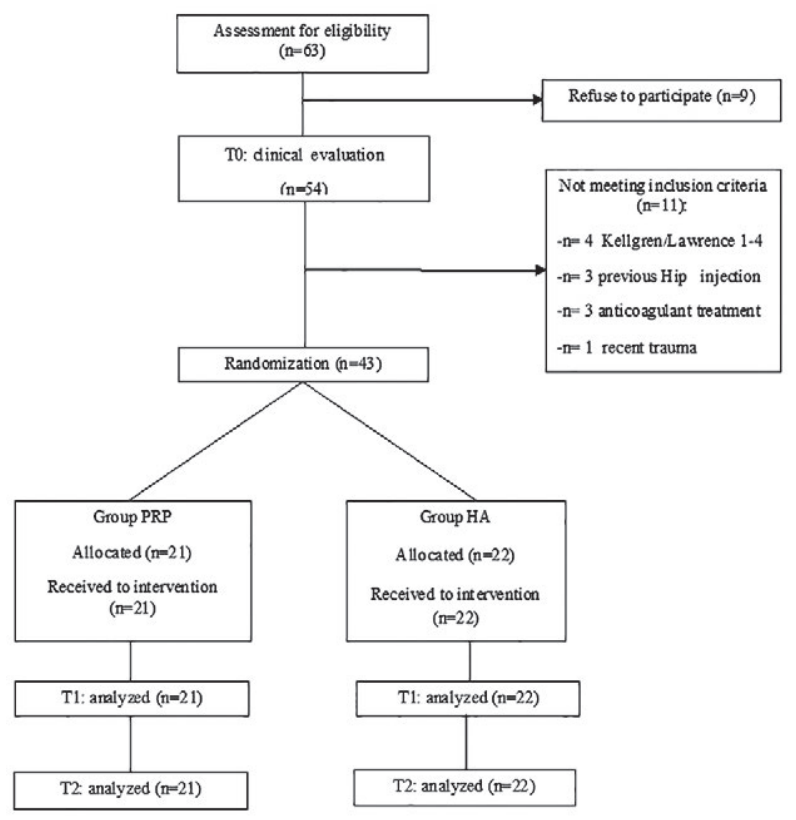

Fig 2. Flow-chart of the patients selection. plied to compare the differences of the baseline data. A 2-way ANOVA with group (experimental versus control) as the between-subjects factor, and time (T0, T1 and T2) as the within-subjects factor was used to detect any significant differences between the experimental and control groups and within each group. A Tukey post-hoc comparison was used to detect any significant differences between the mean values when a significant main effect and interaction were found. The level of significance was set at $\mathrm{p}<0.05$ for all analyses.

\section{Results}

A total of 43 patients were randomized into two groups: HA group $(n=22)$ or PRP group $(n=21)$. Demographic and clinical characteristics of the patients at baseline were well balanced among groups, and are summarized in Table I. No complications related to the IA injections were registered during the treatment and followup period and all patients completed the treatment and performed the post-treatment assessment.

Two-way ANOVA showed a significant group $(\mathrm{F}=5.529 ; \mathrm{p}=0.02)$ and time $(\mathrm{F}=7.491 ; \mathrm{p}=0.001)$ effect for VAS score, and a significant group and time interaction $(\mathrm{F}=5.174 ; \mathrm{p}=0.007)$. Particularly, post hoc analysis revealed that VAS scores were significantly lower than $\mathrm{T} 0$ values at $\mathrm{T} 1$, but not at $\mathrm{T} 2$ in the $\mathrm{PRP}$ group, thereby indicating an immediate effect on pain of PRP which was afterward lost (at T2 VAS value was further reduced but this reduction was not statistically significant).In contrast, in the HA group the significance between VAS values was reached only between $\mathrm{T} 0$ and $\mathrm{T} 2$ values. At $\mathrm{T} 2$, patients in the HA group had lower VAS values than those in the PRP group, the difference being significant at the 2-sample ttest $(\mathrm{p}=0.0004)$. Two-way ANOVA showed a significant group $(\mathrm{F}=32.070 ; \mathrm{p}<0.0001)$ and time $(\mathrm{F}=6.036 ; \mathrm{p}=0.003)$ effect for WOMAC A, while no significant group xtime

Table I. Patient baseline characteristics.

\begin{tabular}{lllll}
\hline & & $\begin{array}{l}\text { Platelet-rich } \\
\text { plasma group } \\
(\mathbf{n = 2 1 )}\end{array}$ & $\begin{array}{l}\text { Hyaluronic } \\
\text { acid group } \\
\mathbf{( n = 2 2 )}\end{array}$ & $\mathbf{p}$ \\
\hline Age (year) & $71.37 \pm 6.03$ & $73.62 \pm 7.87$ & $>0.05$ \\
Sex $\quad$ Female & 10 & 13 & $>0.05$ \\
& Male & 11 & 9 & \\
K-L Grade II & 5 & 7 & $>0.05$ \\
& Grade III & 16 & 15 & $>0.05$ \\
VAS & $6.3(1.56)$ & $7.08(2.04)$ & $>0.05$ \\
WOMAC a & $55.68(21.2)$ & $46.7(22.1)$ & $>0.05$ \\
WOMAC b & $53.7(22.7)$ & $57.6(26.2)$ & $>0.05$ \\
WOMAC c & $59.8(22.5)$ & $45.9(21.7)$ & $>0.05$ \\
\hline
\end{tabular}

K-L: Kellgren and Lawrence scores, VAS: visual-analogue scale, WOMAC: Western Ontario and McMaster Universities index. 
Table II. Visual-Analog-Scale (VAS) and WOMAC scores at baseline (T0), 4-week (T1) and 16-weeks (T2) evaluations in the analysed groups.

\begin{tabular}{|c|c|c|c|c|c|c|}
\hline & \multirow{2}{*}{$\begin{array}{l}\text { Hyaluronic acid group } \\
\text { Mean } \pm \text { SD (95\% CI) }\end{array}$} & \multicolumn{5}{|c|}{ Platelet rich plasma group } \\
\hline & & p-value ${ }^{a}$ & & Mean \pm SD $(95 \%$ CI $)$ & p-value ${ }^{a}$ & \\
\hline \multicolumn{7}{|c|}{ VAS } \\
\hline TO & $6.32 \pm 1.7(5.527-7.113)$ & T0 vs T1 & NS & $7.08 \pm 2.0(6.067-8.100)$ & T0 vs $T 1$ & $<0.01$ \\
\hline$T 1$ & $5.27 \pm 1.6(4.538-6.002)$ & T0 vs T2 & $<0.01$ & $4.73 \pm 3.4(3.016-6.437)$ & T0 vs T2 & NS \\
\hline$T 2$ & $3.63 \pm 2.1(2.624-4.636)$ & $T 1$ vs $T 2$ & NS & $6.36 \pm 2.1(5.307-7.415)$ & $T 1$ vs $T 2$ & NS \\
\hline \multicolumn{7}{|c|}{ WOMAC A } \\
\hline TO & $42.36 \pm 20.5(32.757-51.963)$ & T0 vs T1 & NS & $58.89 \pm 22.0(47.952-69.837)$ & T0 vs T1 & NS \\
\hline$T 1$ & $29.6 \pm 13.4(23.333-35.867)$ & T0 vs T2 & $<0.01$ & $44.27 \pm 28.8(29.964-58.574)$ & $T 0 v s T 2$ & NS \\
\hline$T 2$ & $19.9 \pm 11.4(14.553-25.247)$ & $T 1$ vs $T 2$ & NS & $53.47 \pm 22.3(42.391-64.554)$ & $T 1$ vs $T 2$ & NS \\
\hline \multicolumn{7}{|c|}{ WOMAC B } \\
\hline TO & $57.65 \pm 26.2(45.365-69.935$ & T0 vs T1 & NS & $53.72 \pm 22.7(42.414-65.030)$ & T0 vs $T 1$ & NS \\
\hline$T 1$ & $47.69 \pm 21.2(37.787-57.593)$ & T0 vs T2 & $<0.01$ & $46.42 \pm 27.5(32.738-60.096)$ & T0 vs T2 & NS \\
\hline$T 2$ & $32.91 \pm 20.6(23.249-42.567)$ & $T 1$ vs $T 2$ & NS & $47.22 \pm 22.7(35.936-58.505)$ & $T 1$ vs $T 2$ & NS \\
\hline \multicolumn{7}{|c|}{ WOMAC C } \\
\hline TO & $45.83 \pm 21.7(35.663-55.991)$ & T0 vs $T 1$ & NS & $59.87 \pm 22.5(48.683-71.057)$ & T0 vs T1 & NS \\
\hline$T 1$ & $39.13 \pm 17.2(31.096-47.158)$ & T0 vs T2 & $<0.01$ & $49.13 \pm 29.1(34.675-63.583)$ & $T 0$ vs $T 2$ & NS \\
\hline$T 2$ & $28.39 \pm 17.2(20.360-36.420)$ & T1 vs T2 & NS & $50.80 \pm 22.8(39.480-62.122)$ & $T 1$ vs $T 2$ & NS \\
\hline
\end{tabular}

aBonferroni corrected, NS: not significant, VAS: visual-analogue scale, WOMAC: Western Ontario and McMaster Universities index, SD: standard deviation.

interaction effect was found $(\mathrm{F}=2.488 ; \mathrm{p}=0.09)$. Post hoc analysis revealed that WOMAC A scores were significantly lower than $\mathrm{T} 0$ values at $\mathrm{T} 2$ but not at $\mathrm{T} 1$ in the HA group (Table II). No differences between T0, T1 and T2 values were discernible in the PRP group.

As regards to secondary outcome measures, a significant time ( $\mathrm{F}=4.436 ; \mathrm{p}=0.01)$ effect was found for WOM$\mathrm{AC} B$, while no significant group $(\mathrm{F}=0.471 ; \mathrm{p}=0.49)$ or group xtime interaction $(\mathrm{F}=1.653 ; \mathrm{p}=0.20)$ effects were found. Significant differences at post-hoc analysis were found only in the HA group between T0 and T2 values. A significant group $(\mathrm{F}=14.177 ; \mathrm{p}<0.0001)$ and time $(\mathrm{F}=3.680 ; \mathrm{p}=0.03)$ effect was found for WOMAC $\mathrm{C}$, while no group xtime interaction effect was found $(\mathrm{F}=0.789 ; \mathrm{p}=0.457)$. Again, post-hoc analysis revealed a significant difference between $\mathrm{T} 0$ and $\mathrm{T} 2$ values in the HA group. All these results are detailed in Table II.

\section{Discussions}

The efficacy of PRP IA therapy as compared to HA on pain relief and functional recovery in patients with hip OA was tested in the present study which demonstrated an early effect (4 weeks) of PRP treatment on hip joint pain that however, was not maintained at follow-up. On the contrary, HA produced a long term (16-weeks) pain relief and not a short term response. Interestingly, for all the other outcome measures, no significant effect could be demonstrated.

To our knowledge, only a few studies have aimed at comparing the response to IA treatments with PRP or
HA in hip OA patients. Particularly, a preliminary noncontrolled prospective study supported the safety, tolerability and efficacy of PRP injections for pain relief and improved function in a limited number of patients with OA of the hip [20]. The main difference between that study and our research was the patients' age that was significantly higher in our study.

More recently, ultrasound-guided injection of PRP and HA were used separately and in combination in hip OA patients in a randomized controlled study which demonstrated that intra-articular PRP injections offered a significant clinical improvement in patients with hip OA with a benefit that was significantly more stable up to 12 months. Those results are partially in agreement with our study which showed an early effect of PRP that was not maintained at long term follow-up [19].

Furthermore, the efficacy of ultrasound-guided intraarticular injections of PRP versus HA in hip OA was previously assessed in another study and injections of PRP proved to be efficacious in terms of functional improvement and pain reduction but were not superior to HA in patients with symptomatic hip OA at 12-month follow-up [20]. The results of this study are partially in agreement with our research.

Moreover, the response to multiple PRP injections has been evaluated also in patients with knee degenerative conditions [26] showing a statistically significant improvement on symptoms at 2 and 6 months' follow-up in early knee OA and in the younger subjects group, with the worst results obtained in older patients and especially in 
those with advanced cartilage degeneration. This finding may explain why the PRP injections had only a short term efficacy in our group of patients. Contrarily, IA HA injection demonstrated significant reduction in pain scores, disability scores and analgesic use also in advanced hip $\mathrm{OA}$ [27]. Better results are thus expected in young patients with a low degree of cartilage degeneration treated with HA and that can easily be explained by the mechanism of action hypothesized for the PRP treatment.

The rationale for the use of PRP in tissue regeneration is simply based on the activity of GFs carried in the blood. Autologous PRP is a volume of plasma having platelet concentration above normative baseline values [28]. Depending on the method used to process the PRP, it may also contain white blood cell concentrations above baseline values [29]. Platelets and white blood cells are sources of high concentrations of cytokines, which are well documented as regulating a number of processes related to healing and tissue regeneration [30]. These processes include cell migration, cell proliferation, angiogenesis, inflammation mediation, and collagen synthesis [31]. Several in vitro and in vivo models show that all kinds of cells involved in tissue regeneration are sensitive to growth factors, in particular chondrocytes, osteoblasts and periosteal cell growth is up-regulated by platelet-derived factors such as PDGF and bFGF [30-32] and, what is more, TGF- $\beta$. TGF- $\beta$ is one of the most important factors involved in the process of cartilage regeneration, as it induces chondrogenic differentiation of mesenchymal stem cells as well as matrix deposition [32]. Many studies suggest an important role for these potent biologic regulators of chondrocytes in cartilage repair. Obviously, the potential for GFs action is the presence of a residual number of chondrocytes sensitive to their stimulation. On the other hand, the role of PRP in improving the proliferation of chondrocytes has been recently demonstrated in a controlled laboratory study [33]. Advanced $\mathrm{OA}$ is difficult to treat and presents a challenge for clinicians and orthopedic surgeons because of the distinctive structure and function of hyaline cartilage and its inherently low healing potential [34]. Older and more degenerated joints present a low percentage of living and vital cells and, therefore, a low response potential to the GFs. These considerations are, however, only speculative and hypotheses should be confirmed by studies addressed to understanding the influence of age and cartilage degeneration in response to PRP IA injections.

It should also be noted that in previously performed studies PRP injected into the knees of OA patients was characterized by a high platelets' concentration $(500 \%$ $600 \%$ ) [35]. In our case, the concentration of platelets per milliliter in the PRP represented a mean increase around of $100-150 \%$ compared with whole blood values, and this may have limited the potential therapeutic effect.

The main limitation of the present study is its lack of a control group without therapy. However, we aimed to compare PRP injections directly with HA injections by means of a non-inferiority study, considering the HA injections as a "gold standard" IA therapy [36,37]. The sample size was limited to 43 patients. However, with this sample size, we had a $\beta$ error of at least 0.2 , so that the study demonstrated a good power. Finally, the key point in OA is long-term outcomes ( 1 year to 2 year) as opposed to a 3-month follow-up especially if the mechanism of regeneration is proposed.

In conclusion, the treatment with IA injection of PRP or HA, in elderly patients with OA of the hip, has been proven to be safe and without risks. However, the functional WOMAC and VAS score in the HA has shown to be more effective in the long-term (T2) than the PRP group which presents only significant improvement in VAS scores 4 weeks after treatment (T1). Further studies are needed to confirm these results with longer follow-ups especially if the mechanism of regeneration is proposed and in order to understand the mechanism of action, particular with a view to finding different platelet concentrations and injection timing.

\section{Conflict of interest: none}

\section{References}

1. Poole AR, Rizkalla G, Ionescu M, et al. Osteoarthritis in the human knee: a dynamic process of cartilage matrix degradation, synthesis and reorganization. AgentsActionsSuppl 1993;39:3-13.

2. Iagnocco A, Naredo E. Osteoarthritis: research update and clinical applications. Rheumatology 2012;51(suppl 7):vii2vii5.

3. Bruyn G, Naredo E, Damjanov N, et al. An OMERACT reliability exercise of inflammatory and structural abnormalities in patients with knee osteoarthritis using ultrasound assessment. Ann Rheum Dis 2016;75:842-846.

4. Iagnocco A. Ultrasound in osteoarthritis. Clin Exp Rheumatol 2014;32(1 Suppl 80):S48-S52.

5. Iagnocco A. Imaging the joint in osteoarthritis: a place for ultrasound? Best Pract Res Rheumatol 2010;24:27-38.

6. Migliore A, Tormenta S, Martin Martin LS, et al. The symptomatic effects of intra-articular administration of hylan G-F 20 on osteoarthritis of the hip: clinical data of 6 months follow-up. Clin Rheumatol 2006;25:389-393.

7. Migliore $\mathrm{A}$, Tormenta $\mathrm{S}$, Valente $\mathrm{C}$, et al. Intra-articular treatment with hylan G-F 20 under ultrasound guidance in hip osteoarthritis. Clinical results after 12 months followup. Reumatismo 2005;57:36-43. 
8. Bagga H, Burkhardt D, Sambrook P, March L. Long term effects of intraarticularhyaluronan on synovial fluid in osteoarthritis of the knee. J Rheumatol 2006;33:946-950.

9. Ab-Rahim S, Selvaratnam L, Kamarul T. The effect of TGF-beta1 and beta-estradiol on glycosaminoglycan and type II collagen distribution in articular chondrocyte cultures. Cell Biol Int 2008;32:841- 847.

10. Hickey DG, Frenkel SR, Di Cesare PE. Clinical applications of growth factors for articular cartilage repair. Am J Orthop (Belle Mead NJ) 2003;32:70-76.

11. Loeser RF, Pacione CA, Chubinskaya S. The combination of insulin-like growth factor 1 and osteogenic protein 1 promotes increased survival of and matrix synthesis by normal and osteoarthritic human articular chondrocytes. Arthritis Rheum 2003;48:2188-2196.

12. Schmidt MB, Chen EH, Lynch SE. A review of the effects of insulin-like growth factor and platelet derived growth factor on in vivo cartilage healing and repair. Osteoarthritis Cartilage 2006;14:403-412.

13. Griffin XL, Smith CM, Costa ML. The clinical use of platelet-rich plasma in the promotion of bone healing: a systematic review. Injury 2009;40:158-162.

14. Alsousou J, Thompson M, Hulley P, Noble A, Willett K. The biology of platelet-rich plasma and its application in trauma and orthopaedic surgery. A review of the literature. J Bone Joint Surg Br 2009;91:987-996.

15. Gentile P, De Angelis B, Agovino A, et al. Use of Platelet Rich Plasma and Hyaluronic Acid in the Treatment of Complications of Achilles Tendon Reconstruction. World J Plast Surg 2016;5:124-132.

16. Wang JH, Nirmala X. Application of Tendon Stem/Progenitor Cells and Platelet-Rich Plasma to Treat Tendon Injuries. Oper Tech Orthop 2016;26:68-72.

17. Dines JS, Williams PN, ElAttrache N, et al. Platelet-Rich Plasma Can Be Used to Successfully Treat Elbow Ulnar Collateral Ligament Insufficiency in High-Level Throwers. Am J Orthop (Belle Mead NJ) 2016;45:296-300.

18. Sánchez M, Guadilla J, Fiz N, Andia I. Ultrasound-guided platelet-rich plasma injections for the treatment of osteoarthritis of the hip. Rheumatology 2012;51:144-150.

19. Dallari D, Stagni C, Rani N, et al. Ultrasound-Guided Injection of Platelet-Rich Plasma and Hyaluronic Acid, Separately and in Combination, for Hip Osteoarthritis: A Randomized Controlled Study. Am J Sports Med 2016;44:664-671.

20. Battaglia M, Guaraldi F, Vannini F, et al. Efficacy of ultrasound-guided intra-articular injections of platelet-rich plasma versus hyaluronic acid for hip osteoarthritis. Orthopedics 2013;36:e1501-e1508.

21. Altman R, Alarcón G, Appelrouth D, et al. The American College of Rheumatology criteria for the classification and reporting of osteoarthritis of the hip. Arthritis Rheum 1991;34:505-514.

22. Kellgren JH, Lawrence JS. Radiological assessment of osteo-arthrosis. Ann Rheum Dis 1957;16:494-502.
23. Salaffi F, Leardini G, Canesi B, et al. Reliability and validity of the Western Ontario and McMaster Universities (WOMAC) Osteoarthritis Index in Italian patients with osteoarthritis of the knee. Osteoarthritis Cartilage 2003;11:551-560.

24. Diraçoğlu D, Alptekin K, Dikici F, Balci HI, Ozçakar L, Aksoy C. Evaluation of needle positioning during blind intra-articular hip injections for osteoarthritis: fluoroscopy versus arthrography. Arch Phys Med Rehabil 2009;90:21122115.

25. Tanaka N, Sakahashi H, Sato E, Hirose K, Ishima T, Ishii S. Intraarticular injection of high molecular weight hyaluronan after arthrocentesis as treatment for rheumatoid knees with joint effusion. Rheumatol Int 2002;22:151-154.

26. Kon E, Mandelbaum B, Buda R, et al. Platelet-rich plasma intra-articular injection versus hyaluronic acid viscosupplementation as treatments for cartilage pathology: from early degeneration to osteoarthritis. Arthroscopy 2011;27:14901501.

27. Eyigör C, Pirim A, Eyigör S, Uyar M. Efficacy of intraarticular hyaluronic acid injection through a lateral approach under fluoroscopic control for advanced hip osteoarthritis. Agri 2010; 22:139-144.

28. Pietrzak WS, Eppley BL. Platelet rich plasma: Biology and new technology. J Craniofac Surg 2005;16:1043-1054.

29. Eppley BL, Woodell JE, Higgins J. Platelet quantification and growth factor analysis from platelet-rich plasma: Implications for wound healing. Plast Reconstr Surg 2004;114:1502-1508.

30. Werner S, Grose R. Regulation of wound healing by growth factors and cytokines. Physiol Rev 2003;83:835-870.

31. Nöth U, Rackwitz L, Heymer A, et al. Chondrogenic differentiation of human mesenchymal stem cells in collagen type I hydrogels. J Biomed Mater Res A 2007;83:626-635.

32. Molloy T, Wang Y, Murrell G. The roles of growth factors in tendon and ligament healing. Sports Med 2003;33:381394.

33. Durant TJ, Dwyer CR, McCarthy MB, Cote MP, Bradley JP, Mazzocca AD. Protective Nature of Platelet-Rich Plasma Against Chondrocyte Death When Combined With Corticosteroids or Local Anesthetics. Am J Sports Med 2016. doi: 10.1177/0363546516664161.

34. Alford JW, Cole BJ. Cartilage restoration, part 1: Basic science, historical perspective, patient evaluation, and treatment options. Am J Sports Med 2005;33:295-306.

35. Kon E, Buda R, Filardo G, et al. Platelet-rich plasma: intraarticular knee injections produced favorable results on degenerative cartilage lesions. Knee Surg Sports Traumatol Arthrosc 2010;18:472-479.

36. 37) van den Bekerom MP, Lamme B, Sermon A, Mulier M. What is the evidence for viscosupplementation in the treatment of patients with hip osteoarthritis? Systematic review of the literature. Arch Orthop Trauma Surg 2008;128:815-823.

37. Marshall KW. Intra-articular hyaluronan therapy Foot Ankle Clin 2003;8:221-232. 